\title{
Improved beamforming performance using pulsed plane wave decomposition
}

\author{
Munk, Peter; Jensen, Jørgen Arendt
}

Published in:

Ultrasonics Symposium, 2000 IEEE

Link to article, DOI:

10.1109/ULTSYM.2000.921660

Publication date:

2000

Document Version

Publisher's PDF, also known as Version of record

Link back to DTU Orbit

Citation (APA):

Munk, P., \& Jensen, J. A. (2000). Improved beamforming performance using pulsed plane wave decomposition. In Ultrasonics Symposium, 2000 IEEE (pp. 1749-1754). IEEE. https://doi.org/10.1109/ULTSYM.2000.921660

\section{General rights}

Copyright and moral rights for the publications made accessible in the public portal are retained by the authors and/or other copyright owners and it is a condition of accessing publications that users recognise and abide by the legal requirements associated with these rights.

- Users may download and print one copy of any publication from the public portal for the purpose of private study or research.

- You may not further distribute the material or use it for any profit-making activity or commercial gain

- You may freely distribute the URL identifying the publication in the public portal

If you believe that this document breaches copyright please contact us providing details, and we will remove access to the work immediately and investigate your claim. 


\title{
Improved Beamforming Performance using Pulsed Plane Wave Decomposition.
}

\author{
Peter Munk and Jørgen Arendt Jensen \\ Center for Fast Ultrasound Imaging, Department of Information Technology, Build. 344, \\ Technical University of Denmark, DK-2800 Lyngby, Denmark
}

\begin{abstract}
A tool for calculating the beamformer setup associated with a specified pulsed acoustic field is presented. The method is named Pulsed Plane Wave Decomposition (PPWD) and is based on the decomposition of a pulsed acoustic field into a set of PPWs at a given depth. Each PPW can be propagated to the location of the elements of an array transducer by a time delay. The contribution of each propagated PPW is summed to form one time function for each array element (the BMF matrix). This approach gives the beamformer setup needed to obtain a close approximation to the desired bounded pulsed acoustic field without involving any optimization scheme. The approximation arises due to the limited size of the acoustic aperture and the spatial sampling property of the array transducer. Thus, the acoustical field can be designed according to the imaging needs.

The method is demonstrated by examples in the 2D space by analytical equations, simulation, and experimental results.
\end{abstract}

\section{Introduction}

Traditionally, the plane wave decomposition is referred to as the Angular Spectrum Decomposition. The method is used to propagate fields, but with limited use to backpropagation due to the nature of evanescent waves where the amplitude grows exponentially with the backpropagated distance. The presence of evanescent waves is a consequence of the chosen formalism of the angular spectrum method. The evanescent waves can be eliminated using a projection of the acoustic field most recently described by Leeman and Healey [1], [2]. The projection is carried out as an integration on a plane in the 3D space. A plane projection is an example of the Fourier slice theorem in the theory of reconstruction from projections. In the papers given on this topic it has not been specifically pointed out that the approach assumes local spatial stationarity because time and propagation distance (related through the propagation velocity) have been interchanged in the derivation of the theory. To deal with the related time and space measure, two time measures are introduced. The propagating pulse being evaluated, is frozen at a specific time $t_{0}$, which is referred to as global time, thus a point in time (i.e. depth), where the spatial 3D distribution of the pulse is recorded. This frozen pulse has an oscillatory nature in the propagation direction, which, in a very complex manner, is related to the time signal exciting the transducer. Spatial displacements in the 3D frozen image in the propagation direction of the pulse can be regarded as time assuming local stationarity. This time is referred to as local time $\tau$, which is the time difference to global time $t_{0}$, thus $\tau=t-t_{0}$. A 3D field is decomposed into a set of $1 \mathrm{D}$ projections, where each projection is a time function associated with a plane wave given by the wavenumber $\mathbf{k}=\left(\mathrm{k}_{x}, \mathrm{k}_{y}, \mathrm{k}_{z}\right)$. The projection is carried out as an integration on the $\mathbf{k}$-plane of a time-varying function in the 3D space. The PPWD is developed using the 4D Fourier transform pair. The decomposition makes it possible to propagate a desired pulse shape described in space and time from one location to another. The method is used to decompose a pulsed acoustic field into a set of pulsed plane waves in order to determine an optimized beamformer setup. The use of PPWD gives in details a relationship between the acoustic aperture and the acoustic field. Each PPW is propagated to the elements of an array transducer and is summed to form one time function for each element. These time functions are arranged in a matrix, named the BMF-matrix. This matrix reflects either the required excitations in transmit or the filter impulse responses with delay for receive beamforming. Thus, the individual channel filters will have different impulse responses which implies the need for a filter-and-sum beamformer to obtain proper control of the acoustic field.

\section{Basic theory}

The decomposition method is based on calculation of the spatial Fourier transforms for each temporal frequency. A 3D propagating plane wave, unbounded in space and time, is de- 
scribed by

$$
e(\mathbf{s}, t)=\exp \left(j\left(\omega_{0} t-\mathbf{k}_{0} \cdot \mathbf{s}\right)\right)
$$

where the plane wave is characterized by the wavenumber $\mathbf{k}_{0}$ and the angular frequency $\omega_{0}$. The time is $t$ and $\mathbf{s}$ is the observation point in space. The function (1) is considered to be an elemental function in the 4D Fourier transform pair [3]

$$
\begin{aligned}
p(\mathbf{s}, t) & =\frac{1}{(2 \pi)^{4}} \int_{-\infty}^{\infty} \iiint_{-\infty}^{\infty} P(\mathbf{k}, \omega) \exp (j(\omega t-\mathbf{k} \cdot \mathbf{s})) d \mathbf{k} d \omega \\
P(\mathbf{k}, \omega) & =\int_{-\infty}^{\infty} \iiint_{-\infty}^{\infty} p(\mathbf{s}, t) \exp (-j(\omega t-\mathbf{k} \cdot \mathbf{s})) d \mathbf{s} d t
\end{aligned}
$$

where $\mathbf{k}$ is the wavenumber which determines the propagation direction and, thus, is perpendicular to any iso-phase plane of the wave. Basically (2) shows that any function of space and time can be decomposed in temporal frequency and 3D plane waves. Eqn. (2) is valid for general functions and must be restricted to functions that satisfy the wave equation. An applicable function must meet the condition

$$
\omega^{2}=c^{2}|\mathbf{k}|^{2}=c^{2} k^{2}
$$

which is valid for a lossless medium. An attractive feature of an unbounded harmonic plane wave is that the propagation of such waves can be handled by a simple phase shift. For a pulsed/bounded plane wave, the propagation delay in the $\mathbf{k}$-direction to reach a point $\mathbf{s}_{\mathbf{0}}$ is given by the time

$$
\Delta=\frac{\mathbf{k} \cdot \mathbf{r}}{c}
$$

where $\mathbf{r}$ is the vector from a chosen reference point at the plane wave to $s_{0}$ and $c$ is the propagation velocity.

Thus, in this framework of pulsed plane waves, propagation is handled as a time delay, rather than a phase delay. In order not to alternate the pulse shape, a lossless medium must be assumed.

Using (2), the Fourier transform of $e(\mathbf{s}, t)$ gives

$$
E(\mathbf{k}, \omega)=\delta\left(\mathbf{k}-\mathbf{k}_{\mathbf{0}}\right) \delta\left(\omega-\omega_{0}\right)
$$

which shows that each point in the $(\mathbf{k}, \omega)$ domain corresponds to one unbounded plane wave propagating in the $\mathbf{k}$-direction with an angular temporal frequency of $\omega$. If the plane wave bounded in time is described by

$$
p(\mathbf{s}, t)=a(\mathbf{s}, t) \exp \left(j\left(\omega t-\mathbf{k}_{\mathbf{0}} \cdot \mathbf{s}\right)\right)
$$

the 4D Fourier transform will be

$$
P(\mathbf{k}, \omega)=\delta\left(\mathbf{k}-\mathbf{k}_{\mathbf{0}}\right) \otimes A(\mathbf{k}, \omega)=A\left(\mathbf{k}_{\mathbf{0}}, \omega\right)
$$

The projections are performed on a plane in space with normal vector $\mathbf{k}$, denoted $\mathbf{k}$-plane, and all planes considered rotate around a pivot point ${ }^{1}$ on the axis of propagation. The

\footnotetext{
${ }^{1} \mathrm{~A}$ unique point of rotation shared by all projection planes.
}

coordinate system $\mathbf{s}=(x, y, z)$ is chosen to have the $z$-axis parallel to the propagation direction of the acoustic pulse and $k_{x}$ and $k_{y}$ and $k_{z}$ are the components of the normal vector $\mathbf{k}$ along the $x-, y-, z$-axes respectively. For plane waves, an isophase surface in space is a $3 \mathrm{D}$ plane with a constant value. It defines the wavefront, and the surface can be described as $\mathbf{k} \cdot \mathbf{s}=k_{x} x+k_{y} y+k_{z} z=$ constant.

A $1 D$ signal is calculated for the propagating pulse passing through the projection plane over which the integration is performed. Performing an integral over a k-plane results in a time function describing the pulse shape for this particular plane wave propagating in the $\mathbf{k}$-direction. Thus, for each value of $\mathbf{k}$ there is a local time function $P(\mathbf{k}, \tau)$ as a result of the surface integration. Compared to previous work of Leeman and Healey, this approach incorporates time dependence in the directivity function, named $P(\mathbf{k}, \tau)$. Here the time is local time. The propagation of the bounded plane wave is performed in the global time domain on $P(\mathbf{k}, \tau)$ according to the time delay from one location of the axis of the propagation direction to the new location. This approach is referred to as pulsed plane wave decomposition and is closely related to the Leeman approach. The key issue is the use of plane wave projections and the 0 of global and local time.

The orientation of the integration plane is given by two angles $\theta$ and $\phi$. A new rectangular coordinate system, rotated relative to first one, is introduced in order to describe the mathematical foundation of the calculation method. One coordinate system is used to describe the propagating pulse and the other to describe the integration over the $\mathbf{k}$-plane. A rotation of the coordinate system is used to align the new coordinate system to the $\mathbf{k}$-plane. The coordinates change from $\mathbf{s}$ to $\mathbf{s}^{\mathbf{k}}$. The origin of the coordinate systems is the pivot point of the integration plane. With the proper choice of angle definitions, the Jacobian for the coordinate rotations will be one, i.e. $d x d y d z=d x^{k} d y^{k} d z^{k}$.

This relation makes it possible to perform an integration with respect to a coordinate system that follows the wave number of the integration plane.

The integration over the plane, with reference to the rotated coordinate system, is given by

$$
P_{(p)}\left(z^{k}, t\right)=\iint_{-\infty}^{\infty} d x^{k} d y^{k} p\left(x^{k}, y^{k}, z^{k}, t\right) .
$$

The new coordinate system $\mathbf{s}^{\mathbf{k}}=\left(x^{k}, y^{k}, z^{k}\right)$ is chosen to have the $z^{k}$-axis parallel to the normal vector $\mathbf{k}$. The new coordinate system simplifies the plane integral and the result is an projection onto the $\left(z^{k}, t\right)$-plane. A relation between $P(\mathbf{k}, t)$ and the projection $P_{(p)}\left(z^{k}, t\right)$ will be established in the following.

In the context of pulsed propagating plane waves only the 3 D spatial Fourier transform must be considered, because the global time dimension is fixed at $t_{0}$. Thus, (2) becomes 


$$
\begin{aligned}
& p\left(\mathbf{s}, t_{0}\right)=\frac{1}{(2 \pi)^{3}} \iiint_{-\infty}^{\infty} P\left(\mathbf{k}, t_{0}\right) \exp \left(j\left(\omega t_{0}-\mathbf{k} \cdot \mathbf{s}\right)\right) d \mathbf{k} \\
& P\left(\mathbf{k}, t_{0}\right)=\iiint_{-\infty}^{\infty} p\left(\mathbf{s}, t_{0}\right) \exp \left(-j\left(\omega t_{0}-\mathbf{k} \cdot \mathbf{s}\right) d \mathbf{s} .\right.
\end{aligned}
$$

The local time $\tau$, i.e. a displacement $\varepsilon=c \tau$ in the propagation direction $z$, is introduced as a third variable relative to the position $\mathbf{s}$

$$
\begin{aligned}
& p\left(\mathbf{s}, t_{0}, \tau\right)=\frac{1}{(2 \pi)^{3}} \iiint_{-\infty}^{\infty} P\left(\mathbf{k}, t_{0}, \tau\right) \exp \left(j\left(\omega t_{0}-\mathbf{k} \cdot \mathbf{s}\right)\right) d \mathbf{k} \\
& P\left(\mathbf{k}, t_{0}, \tau\right)=\iiint_{-\infty}^{\infty} p\left(\mathbf{s}, t_{0}, \tau\right) \exp \left(-j\left(\omega t_{0}-\mathbf{k} \cdot \mathbf{s}\right) d \mathbf{s} .\right.
\end{aligned}
$$

$P\left(\mathbf{k}, t_{0}, \tau\right)$ is the time function that describes the bounded pulsed plane wave with the wave number vector $\mathbf{k}$ at a specific time instant $t_{0}$. Equation (10) describes a pulsed decomposition of any pulsed wave field. Using (10) in (8) the plane integral becomes

$$
\begin{aligned}
P_{(p)}\left(z^{k}, t_{0}, \tau\right) & =\frac{1}{(2 \pi)^{3}} \iint_{-\infty}^{\infty} d x^{k} d y^{k} \\
& \cdot \iiint_{-\infty}^{\infty} P\left(\mathbf{k}, t_{0}, \tau\right) \exp \left(j\left(\omega t_{o}-\mathbf{k} \cdot \mathbf{s}\right)\right) d \mathbf{k} .
\end{aligned}
$$

The local time $\tau$, which is in the $z$-direction, can be written as an exponential in the Fourier integral, and writing the vector in coordinate components gives

$$
\begin{aligned}
P_{(p)}\left(z^{k}, t_{0}, \tau\right) & =\frac{1}{(2 \pi)^{3}} \iint_{-\infty}^{\infty} d x^{k} d y^{k} \\
& \cdot \iiint_{-\infty}^{\infty} d k_{x}^{k} d k_{y}^{k} d k_{z}^{k} P\left(k_{x}^{k}, k_{y}^{k}, k_{z}^{k}\right) \\
& \cdot \exp \left(-j\left(k_{x}^{k} x^{k}+k_{y}^{k} y^{k}+k_{z}^{k} z^{k}+\omega \tau+\omega t_{0}\right)\right) .
\end{aligned}
$$

Using the definition of the delta function by its Fourier integral and

$$
\omega=k_{z} c=k_{z}^{k} \frac{c}{\cos \theta \cos \phi}=k_{z}^{k} c^{k}
$$

the plane integration becomes

$$
\begin{aligned}
P_{(p)}\left(z^{k}, t_{0}, \tau\right) & =\frac{1}{2 \pi} \int_{-\infty}^{\infty} d k_{z}^{k} P\left(0,0, k_{z}^{k}\right) \\
& \cdot \exp \left[j k_{z}^{k}\left(z^{k}-c^{k} \tau-c^{k} t_{0}\right)\right]
\end{aligned}
$$

in which $P\left(0,0, k_{z}^{k}\right)$ indicates the collapse of two dimensions, the $x$ and $y$ direction, changing the integration over $\mathbf{k}$ to the $k_{z}$-direction. The one projection $P_{(p)}$ can be written as a displacement to a location corresponding to $t_{1}$ as

$$
P_{(p)}\left(z^{k}, t_{1}, \tau\right) \equiv P_{(p)}\left(z^{k}-c^{k}\left(\tau+t_{0}-t_{1}\right), t_{0}, 0\right) .
$$

If a pulsed field, given at a time instant $t_{0}$, has been decomposed into a set of pulsed plane waves, each of these plane waves can be propagated to any location in space and the pulsed wave field can be constructed at a new location. Here it must be emphasized that the velocity $c_{k}$ used to propagate the plane wave $\mathbf{k}$ is adjusted according to the angle between the propagation direction $z$ and the normal vector $k$ (equal to $z^{k}$.

\section{Results}

Analytical equations, simulation and experimental results are given to verify and demonstrate the method. The choice is directly related to the acoustic fields used for vector velocity estimation [4]. An analytical solution to the plane wave decomposition is carried out in 2D in the $x z$-plane. A 2D Gaussian function forms the envelope of the specified PSF with a harmonic oscillation in either one dimension (axial) or two dimensions (axial and lateral). The projections of the PPWD are calculated and propagated analytically, and summed numerically for each array element to form the BMF matrix. First, the BMF matrix is used as the excitations in a simulation using Field II [5] to compare the desired and the simulated field. Second, the BMF matrix is used in an experimental study as time filters in receive mode to create a desired sensitivity function. The measurement was made in a water tank.

\subsection{Simulation}

The time is fixed at $t_{0}$ and the point spread function is described in $x z$-space. The envelope of the PSF is defined by a 2D Gaussian function where the standard deviations determine the axial and the lateral resolution. The functions limited by the envelope are harmonic functions. Either one dimensional, in the axial direction, or two dimensional, in the axial and lateral direction. These acoustic fields are analogous to the ones used for measurement of the velocities in either $1 \mathrm{D}$ or $2 \mathrm{D}$, respectively.

The examples will operate only on two spatial dimensions, the lateral $x$ - and the axial $z$-direction. The reduction from 3 to 2 dimensions causes the plane integral to become a line integral. The angle between the line of integration and the axis of propagation, $z$, is denoted $\theta$. The integration line is named $\theta$-line.

The two pulses considered are

- case 1: a conventional Gaussian pulse oscillating in the axial direction with the frequency $f_{0}$, see Fig. 1

- case 2: a 2D double oscillating field with an axial and a lateral/transverse oscillation. The lateral frequency is $f_{x}$ and has the unit of $\left[\mathrm{m}^{-1}\right]$, see Fig. 2 


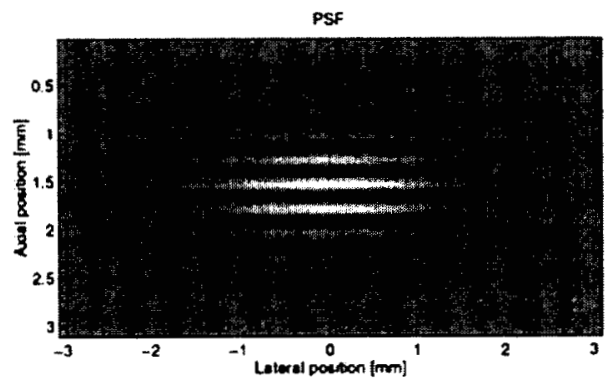

Figure 1: Conventional Gaussian enveloped pulsed pressure field

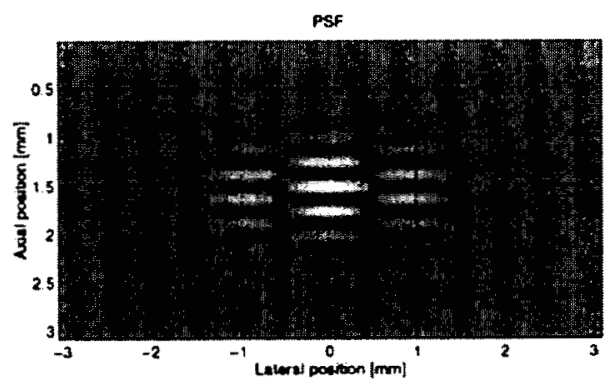

Figure 2: Double oscillating Gaussian enveloped pulsed pressure field

The conventional Gaussian pulse is a special case of the double oscillating field. The oscillation function is denoted $f(x, z, t)$. In both cases the pulse is bounded in the axial and lateral direction by a 2D Gaussian envelope function $g(x, z, t)$. The extend of the pulse is controlled by the axial standard deviation $\sigma_{z}$ and the lateral standard deviation $\sigma_{x}$. The oscillating function is

$$
\begin{aligned}
f(x, z, t) & =\cos \left(2 \pi f_{x} x\right) \cos \left(2 \pi f_{0} \frac{z}{c}-2 \pi f_{x} x\right) \\
& =\frac{1}{2} \cos \left(2 \pi f_{0}\left(\frac{z}{c}-t\right)-2 \pi f_{x} x\right) \\
& +\frac{1}{2} \cos \left(2 \pi f_{0}\left(\frac{z}{c}-t\right)+2 \pi f_{x} x\right) \\
& =f_{1}(x, z, t)+f_{2}(x, z, t)
\end{aligned}
$$

and the envelope function is

$g(x, z, t)=\frac{1}{2 \pi} \frac{1}{\sigma_{x} \sigma_{z}} \exp \left(-\frac{1}{2}\left[\left(\frac{x}{\sigma_{x}}\right)^{2}+\left(\frac{z-c t}{\sigma_{z}}\right)^{2}\right]\right)$.

With this description, there is a geometric relation between $x$ and $z$, which gives the shape of the pulse, and a time dimension which allows propagation of the pulse in local time. The field sensitivity is given by $h(x, z, t)=f(x, z, t) g(x, z, t)$ and the line integral is denoted

$$
H(t ; \boldsymbol{\theta})=\int_{\boldsymbol{\theta}-\text { line }} h(x, z, t) d x d z .
$$

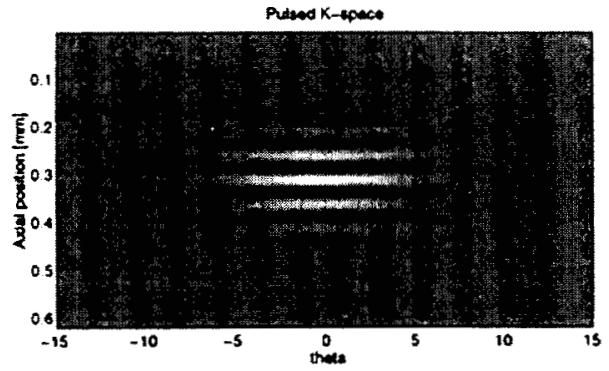

Figure 3: Pulsed K-space for conventional Gaussian pulse.

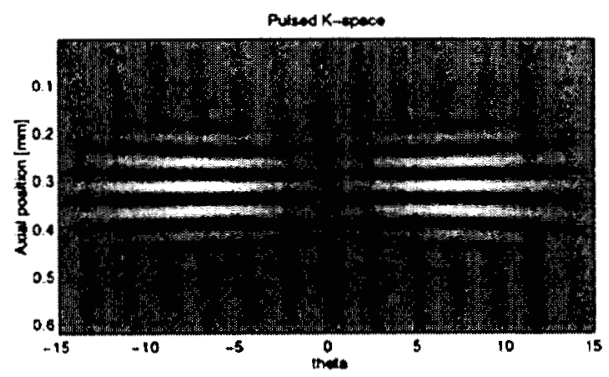

Figure 4: Pulsed K-space for double oscillating Gaussian pulse.

Thus, the pressure function in the $x z$-plane is decomposed in the 2D-space by line integrals to a number of time functions. A line in the $x z$-space is given by $z=x \tan \theta$, which is used for making the integral dependent on one variable $x$. An analytical solution to the line integration with $\boldsymbol{\theta}$ as a parameter is given. The function $h(x, z, t)$ is divided in two functions $h(x, z, t)=h_{1}(x, z, t)+h_{2}(x, z, t)$ and the result $H_{n}(t ; \theta)$, with $n=1,2$, becomes

$$
\begin{array}{cc}
H_{n}(t ; \theta)=\frac{1}{4 \pi} \frac{1}{\sigma_{x} \sigma_{z}} \sqrt{\frac{\pi}{a}} \exp \left(\frac{b^{2}}{a}-d-\frac{q_{n}^{2}}{a}\right) \cos \left(-2 \frac{b}{a} q_{n}+r\right) \\
a=\frac{1}{2}\left(\frac{1}{\sigma_{x}^{2}}+\frac{\tan ^{2} \theta}{\sigma_{z}^{2}}\right) & d=\frac{c^{2} t^{2}}{2 \sigma_{z}^{2}} \\
q_{1}=\pi \frac{f_{0}}{c} \tan \theta-\pi f_{x} & b=-\frac{a t \tan \theta}{2 \sigma_{z}^{2}} \\
q_{2}=\pi \frac{f_{0}}{c} \tan \theta+\pi f_{x} & r=-2 \pi f_{0} t .
\end{array}
$$

The final solution is

$$
H(t ; \theta)=H_{1}(t ; \theta)+H_{2}(t ; \theta)
$$

The pulsed K-space for the two examples is shown in Fig. 3 and Fig. 4. The abscissa is the angle between a line parallel to the $x$-axis and the integration line. The ordinate is the axial dimension of the pulsed plane wave. There is one time function for each angle $\theta$.

Now the plane wave distribution $H(t ; \theta)$ is known and the individual pulsed plane waves can be propagated to an acoustic aperture at any location in the $x z$-plane in order to evaluate the needed spatial distributed excitation function. The 


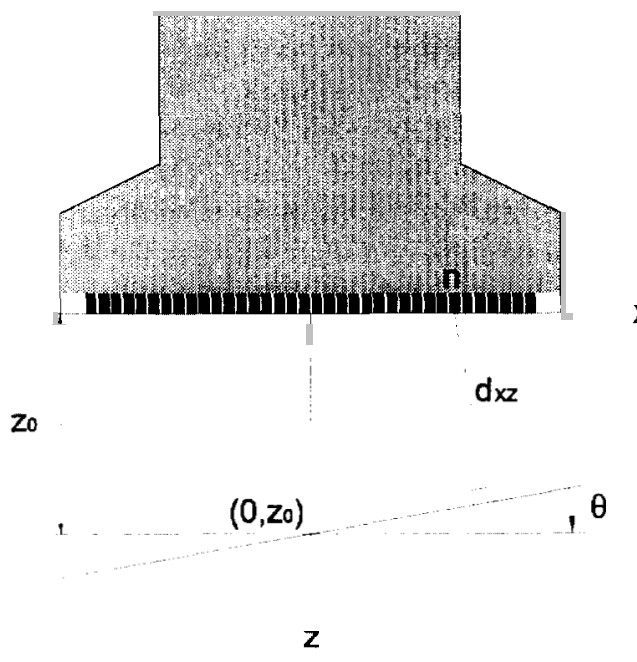

Figure 5: Schematics for delay calculation applied for backpropagation.

distance $d_{x z}$ from a given integration line to an element on a linear array transducer, see Fig. 5 for the schematics, is

$$
d_{x z}\left(\theta, z_{0}, n\right)=\frac{p\left(n-\frac{1}{2}\right) \tan \theta+z_{0}}{\sqrt{\tan ^{2} \theta+1}}
$$

where $n$ is the element number, $p$ is the pitch ${ }^{2}, z_{0}$ is the rotation point on the $z$-axis, $\theta$ is the angle of the plane wave considered. The velocity applied to each of the $\theta$-plane waves is $c_{\theta}=\frac{c}{\cos \theta}$. The propagation is done for the double oscillating case, and the acoustic aperture is a 128 element array transducer primarily defined by pitch $0.156 \mathrm{~mm}$. Fig. 6 shows the resulting excitation for each element.

The matrix has one column for each time sample. The size of the column corresponds to the number of array elements. Thus, each row is the time function associated with an element. The calculated element excitation is used for a simulation using Field II to make a comparison of the desired field with the simulated field. The result can be seen in Fig. 7 .

The result shows that it is possible to analytically decompose a pulsed field into pulsed plane waves, propagate these waves to the positions of the array elements, sum up the contribution for all plane waves at the individual element, and use the analytical result of the summed pulses as an excitation for the elements of an array transducer to obtain the desired acoustic field. Fig. 7 shows two pulsed fields which are in close agreement. The top field is the desired field and

\footnotetext{
${ }^{2}$ Pitch is the center to center distance for two adjacent elements in an array transducer
}

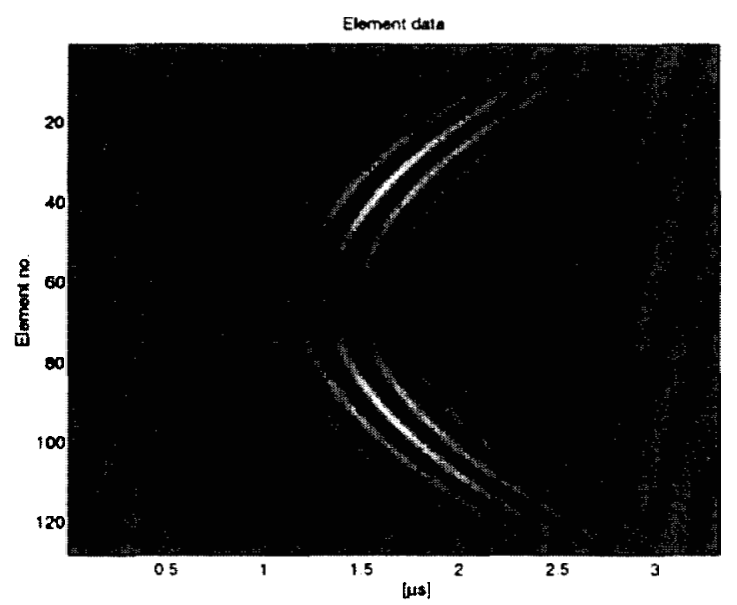

Figure 6: BMF matrix for the array transducer, one row for each element.

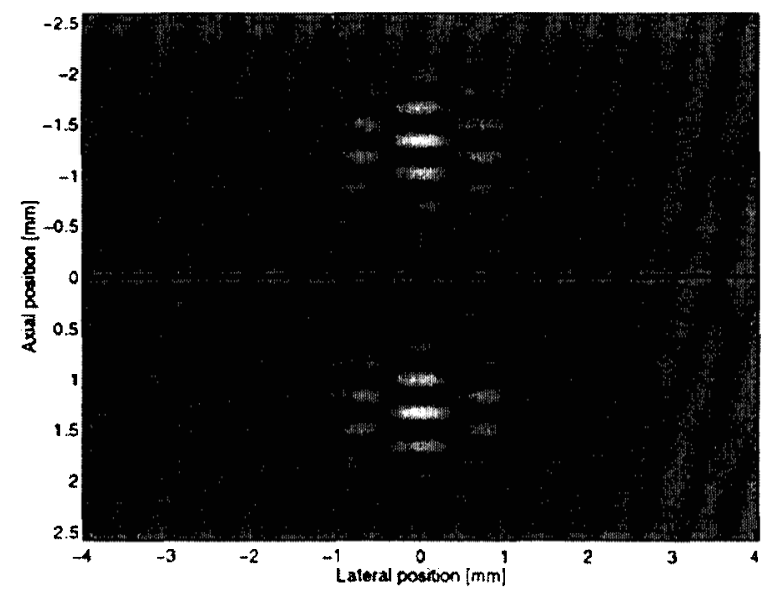

Figure 7: Comparison between the desired (top) field and the decomposed-backpropagated-transmitted field.

the bottom field is the beamformed field. The lateral oscillation has the correct period and the part of the simulated field, which double oscillates, resembles the original desired field. The RMS error is found to be $1.6 \%$.

\subsection{Experiment}

The experimental results were obtained using a 1/64" ruby as a point reflector. The ruby was moved horizontally approximately parallel to the transducer surface. For each position of the point reflector, $128 \mathrm{RF}$-lines were recorded (one for each channel of the scanner) and all data were saved based on channel data. The recorded data are post beamformed for each position of the point reflector. For each position the beamformed RF-line is saved and finally the 2-D response from one depth is created. The new BMF matrix was used 


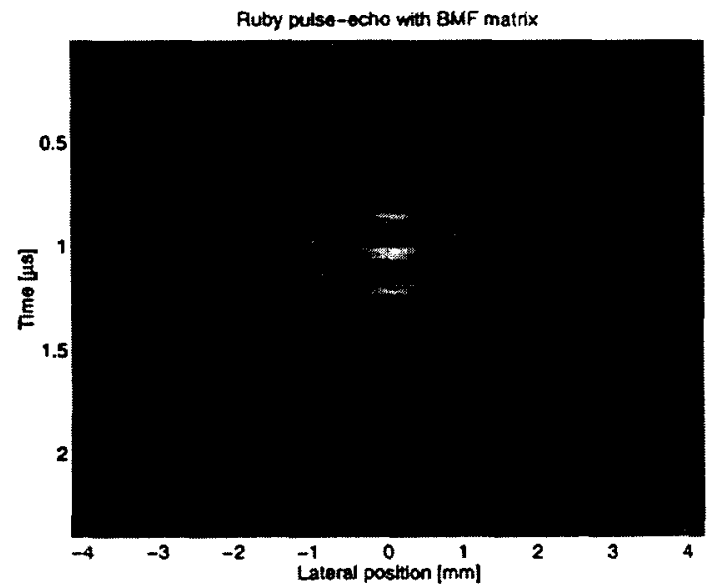

Figure 8: Pulse echo PSF of experimental data from ruby using the BMF matrix.

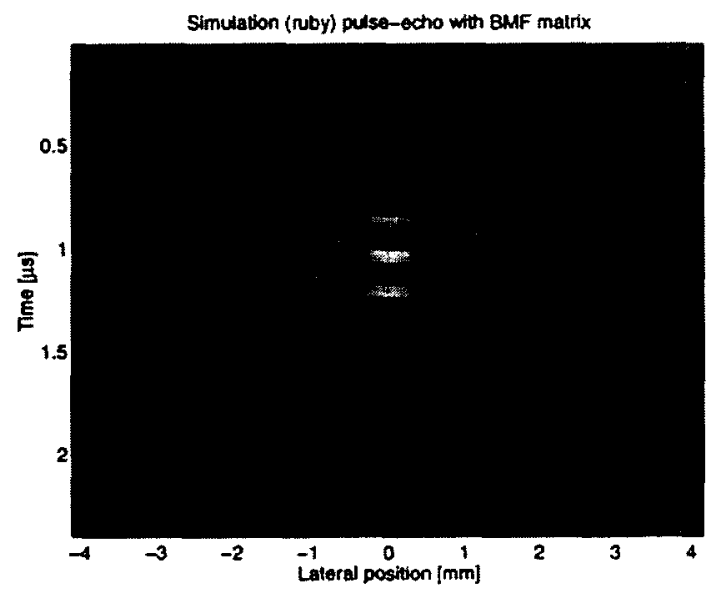

Figure 9: Pulse echo PSF of simulated data using the BMF matrix.

as a spatial and temporal matched filter in the receive beamforming and the result is shown in Fig. 8

For comparison the results of a corresponding simulation is shown in Fig. 9

Due to mechanical misalignment in the measurement it is not feasible to calculate a RMS error. A qualitatively evaluation can be done by graphical comparison.

Compared to previous results [6], this example demonstrates the ability of the PPWD to form a BMF matrix which improves the control of the field sensitivity. In this case the sidelobe energy has been reduced.

\section{Conclusion}

The PPWD presented in this paper gives a tool to relate an acoustical field to a beamformer setup without involving any optimization scheme. With this method a desired pulse at a given time $t_{0}$ can be decomposed into pulsed plane waves. Each of these plane waves can be propagated to each of the elements of an array transducer. The contribution of each propagated pulsed plane wave to a transducer element is summed up resulting in the BMF matrix. The BMF matrix can be used as the excitation for the array transducer to create the original desired pulse or as time filters in the receive beamforming. The usefulness of the PPWD is demonstrated by an analytical PPWD of a 2D Gaussian pulse. The calculated BMF matrix is used for transmit in an simulation and the resulting field is in very close agreement with the initial mathematical formulated field. The receive beamforming was demonstrated on the basis of experimental data which was compared to a corresponding simulation.

\section{Acknowledgment}

This work was supported by grant 9700883 and 9700563 from the Danish Science Foundation and by B-K Medical A/S, Gentofte, Denmark and by grant EF-632 from the Danish Academy of Technical Sciences. The authors are greatful Dr. Gregg Trahey and Dr. Martin Anderson at Duke University, NC, USA for providing the measured data used in this paper.

\section{References}

[1] S. Leeman and A. J. Healey. Field propagation via the angular spectrum method. In Acoust. Imaging, volume 23, pages 363-368, 1997.

[2] A. J. Healey and S. Leeman. Space-time imaging of transient ultrasound fields. International Journal of Imaging Systems and Technology, 8(1):45-51, 1997.

[3] D. E. Dudgeon. Multidimensional Digital Signal processing. Prentice-Hall, first edition, 1984.

[4] P. Munk. Estimation of the 2-D flow vector in ultrasonic imaging: a new approach. Master's thesis, Deparment of Information Technology, Technical University of Denmark, 1996.

[5] J. A. Jensen. Field: A program for simulating ultrasound systems. Med. Biol. Eng. Comp., 10th Nordic-Baltic Conference on Biomedical Imaging, Vol. 4, Supplement 1, Part 1:351-353, 1996b.

[6] P. Munk and J. A. Jensen. Performance of a vector velocity estimator. In Proc. IEEE Ultrason. Symp., pages 1489-1493, 1998. 